\title{
SOLVING THE JOB-SHOP SCHEDULING PROBLEM WITH A SIMPLE GENETIC ALGORITHM
}

\author{
Lestan, Z.; Brezocnik, M.; Buchmeister, B.; Brezovnik, S. \& Balic, J. \\ University of Maribor; Faculty of Mechanical Engineering, \\ Smetanova 17, SI - 2000 Maribor, Slovenia \\ E-Mail: zoki2905@gmail.com
}

\begin{abstract}
The job-shop scheduling is concerned with arranging processes and resources. Proper schedules are very important for the manufacturers, but can cause serious problems because of the enormous solution space. Pressure from the competitive enterprises is the main reason why time is becoming one of the most important success factors. Scheduling tools allow production to run efficiently. The goal in this paper is the development of an algorithm for the job-shop scheduling problem, which is based only on genetic algorithms. Our intention is to prove, that even a relatively simple genetic algorithm is capable for job-shop scheduling. The effectiveness of the algorithm is demonstrated by solving practical problems. The first problem consists of $10 \times 10$ instances ( 10 jobs and 10 machines) and the second one of $20 \times 5$ instances (20 jobs and 5 machines). The scheduling efficiency is measured by the time required to complete all jobs (makespan). In case of the first and the second problem, the best obtained solution (i.e., deviation from optimal solution) was $1.2 \%$ and $4 \%$, respectively. (Received in March 2009, accepted in June 2009. This paper was with the authors 1 month for 1 revision.)
\end{abstract}

Key Words: Job-Shop Scheduling, Manufacturing, Genetic Algorithms, Evolutionary Computation

\section{INTRODUCTION}

Scheduling is an act of defining priority or arranging activities to meet certain requirements, constraints, or objectives. A schedule is a timetable for both jobs and machines. Time is a major constraint and we must utilise it in an optimum manner. Scheduling the production resources leads to increased efficiency, utilisation and profitability for the enterprise.

Job-shop is one of the most popular generalized production systems. Because of the challenging nature of the problem, many researchers have analysed job-shop scheduling. It belongs to the most intractable problems considered.

In the job-shop problem (with no recirculation) a set of jobs $J=\left(J_{1}, J_{2}, \ldots, J_{n}\right)$ must be processed on a set of machines $M=\left(M_{1}, M_{2}, \ldots, M_{m}\right)$, where $n$ is the number of jobs and $m$ is the number of machines. Each job consists of a sequence of operations, where each operation must be processed on a predefined machine for an exact time [1-4]. All operations of a job must be processed one after another in the given order. We assume that there is sufficient buffer space between the machines to store a job if it finishes on one machine and the next machine is still occupied by another job. There are several constrains on jobs and machines $[5,6]$ :

- there are no precedence constrains among operations of different jobs,

- operations cannot be interrupted,

- each machine can process only one job at a time,

- a job does not visit the same machine twice,

- neither release times nor due dates are specified. 
The objective is to minimize the makespan, i.e., the maximum of job completion times. In order to do this, the right operation sequences on the machines have to be determined.

The job-shop problem is a problem in discrete or combinatorial optimization, and is a generalization of the well-known travelling salesman problem. It is a very good illustration of a class of problems in computational complexity theory which are hard to solve. If we already know that the travelling salesman problem is NP-hard (as it is), then the job-shop problem is clearly also NP-hard, since the travelling salesman problem is the job-shop problem with only one machine $m=1$ (the salesman is the machine and the cities are the jobs). In computational complexity theory, NP ("Non-deterministic Polynomial time") is the set of decision problems solvable in polynomial time on a non-deterministic Turing machine.

Because the job-shop scheduling problem is a NP-hard problem, it has captured the interest of a significant number of scientists, so a lot of algorithms have been proposed for solving it [7]. The algorithms which base on the method branch and bound are useful only for solving small instances. Instances with a larger number of operations cannot be solved in a reasonable time. For large scale problems approximation algorithms are used: shifting bottleneck, genetic algorithm, simulated annealing, tabu search, priority dispatch, etc. Although it is not possible to solve even modest sized instances to optimality, a number of important algorithmic advances have been made in the past few years [8]. The latest studies indicate that the best results are obtained with the use of hybrid methods involving genetic algorithms, tabu search, simulated annealing and the shifting bottleneck approach. One of the most promising methods for job-shop scheduling is a hybrid method based on simulated annealing and tabu search [9]. The advantage of tabu search is in a memory function which prevents that the search would end in a local optimum. If this method is combined with the method which is analogue to physical annealing and independent from the initial solution, a very effective search algorithm is made. Similar approaches could be also used for solving many similar optimization problems in other fields (see for example [10-13]).

In this paper a simple genetic algorithm is used to treat the job-shop problem. This algorithm was developed independently, without regard for the work of other researchers. The intention was to make a simple algorithm which will try to find the schedule with the smallest makespan. Only genetic operations are used in order to achieve this. It is possible to schedule a various number of jobs, but neither release times nor due dates are considered. Only selection and permutation are used as genetic operations. The algorithm uses random moves to search for the optimal schedule in the solution space, which means that the solution is obtained without the help of heuristic methods. This paper is organized as follows: in Section 2 , the encoding method used in the algorithm is introduced. Section 3 describes the evaluation of encoded solutions, in Section 4, the genetic operations are described, in Section 5, the influence of evolution parameters on the search procedure is explained, in Section 6, two experimental results are shown and in Section 7 is the conclusion.

\section{ENCODING}

How to encode solutions to chromosomes to ensure feasible solutions is a key issue for genetic algorithms. Researchers use different representations for the job-shop scheduling problem [14]:

- operation-based representation,

- machine-based representation,

- disjunctive graph-based representation,

- preference list-based representation,

- job-based representation,

- priority rule-based representation. 
In our algorithm, the preference list-based representation is used. In this encoding method the operations are arranged in a certain order. It depends on this order, how the operations will be processed on the machines. It is very important, that the precedence constrains of operations of individual jobs are considered. This means, that the sequence of operations of a job must stay intact also in the encoded solution. How the encoding works is shown on a simple example. Table I shows a $3 \times 3$ instance; 3 jobs ( 9 operations) must be scheduled on 3 machines $(M 1, M 2, M 3)$ to achieve the smallest possible makespan.

Table I: $3 \times 3$ instance.

\begin{tabular}{|c|c|c|c|c|c|c|}
\hline \multirow{2}{*}{ Jobs } & \multicolumn{3}{|c|}{ Processing times } & \multicolumn{3}{c|}{ Processing order } \\
\cline { 2 - 7 } & \multicolumn{3}{|c|}{ operations } & \multicolumn{3}{c|}{ operations } \\
\cline { 2 - 7 }$J 1$ & 29 & 78 & 9 & $\begin{array}{c}\text { machine } \\
M 1\end{array}$ & $\begin{array}{c}\text { machine } \\
M 2\end{array}$ & $\begin{array}{c}\text { machine } \\
M 3\end{array}$ \\
\hline J2 & 43 & 90 & 28 & $\begin{array}{c}\text { machine } \\
M 1\end{array}$ & $\begin{array}{c}\text { machine } \\
M 3\end{array}$ & $\begin{array}{c}\text { machine } \\
\text { M2 }\end{array}$ \\
\hline$J 3$ & 91 & 85 & 74 & $\begin{array}{c}\text { machine } \\
M 2\end{array}$ & $\begin{array}{c}\text { machine } \\
M 1\end{array}$ & $\begin{array}{c}\text { machine } \\
M 3\end{array}$ \\
\hline
\end{tabular}

From the Table I it is possible to write the operation sequence for each job:

$$
\begin{aligned}
& J 1 \text { (J1 M1 29) (J1 M2 78) (J1 M3 9) } \\
& \text { J2 (J2 M1 43) (J2 M3 90) (J2 M2 28) } \\
& \text { J3 (J3 M2 91) (J3 M1 85) (J3 M3 74) }
\end{aligned}
$$

Job $J 1$ must first be processed on machine $M 1$ for 29 time units. After that on machine $M 2$ for 78 units and the last is machine $M 3$ for 9 units. Similar goes for the other two jobs. The schedule for this instance is encoded into a string, where the position of the operation in the string plays an important role. Operations are ordered with the help of a randomizer. Therefore it is important to use a reliable randomizer. String making in our case looks like this:

a) A list of first operations of all jobs is made.

$$
\text { ((J1 M1 29) (J2 M1 43) (J3 M2 91)) }
$$

b) From the list of first operations, one operation is chosen randomly; let's say (J2 M1 43). This operation is the first operation in the string. The operation is taken from the corresponding job and inserted into the string.

$$
\begin{aligned}
& \text { J1 (J1 M1 29) (J1 M2 78) (J1 M3 9) } \\
& \text { J2 (J2 M3 90) (J2 M2 28) } \\
& \text { J3 (J3 M2 91) (J3 M1 85) (J3 M3 74) }
\end{aligned}
$$

String:

$$
\text { ((J2 M1 43)) }
$$

c) Again a list of first operations of all the jobs is made and an operation is randomly selected from the list; let's say ( $J 1$ M1 29). This operation is taken from the corresponding job and inserted as second operation in the string. 


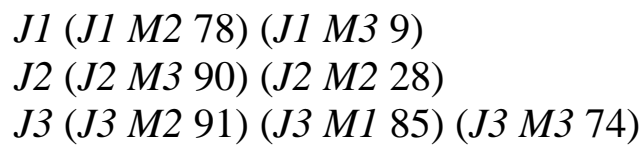

String:

((J2 M1 43) (J1 M1 29))

d) The procedure is repeated until all the operations from the jobs are transferred into the string.

If the procedure, described above, would be continued until the end, the string could look like this:

String:

((J2 M1 43) (J1 M1 29) (J1 M2 78) (J3 M2 91) (J2 M3 90) (J3 M1 85) (J2 M2 28) (J1 M3 9) (J3 M3 74))

This string will be used later on for demonstrations. A closer look at the string reveals that the precedence constrains have been considered during the making of the string. Job operations in the string still have the same processing order, but now they are mixed together. Why this is so important is explained in the next section.

Because the string making is left to coincidence, it is possible to make a lot of versatile strings (organisms) which are necessary for the initial population.

\section{EVALUATION}

Only feasible strings represent a solution to our problem and therefore it is very important that feasibility is maintained throughout the searching process. Because in our case the goal lies in the time optimization of schedules, we are interested in the makespan. Besides the makespan we are interested also in the processing order on the machines. For that reason, Gantt charts are used for string evaluation. On the $\mathrm{y}$ - axis of the Gantt chart the operations are applied and the $\mathrm{x}$ - axis represents the time. The Gantt chart (string evaluation) is done step by step with adding operations one after another. In our case the operations are added directly from the string, from the left side to the right side. The operations which are at the beginning of the string have a higher processing priority than those at the end. This means, that the Gantt chart and the makespan depend only upon the order in the string. That is why it is so important, that the operation order in the string is according to the precedence constrains. Otherwise the evaluation would give a false value. The Gantt chart for our string is shown in Fig. 1.

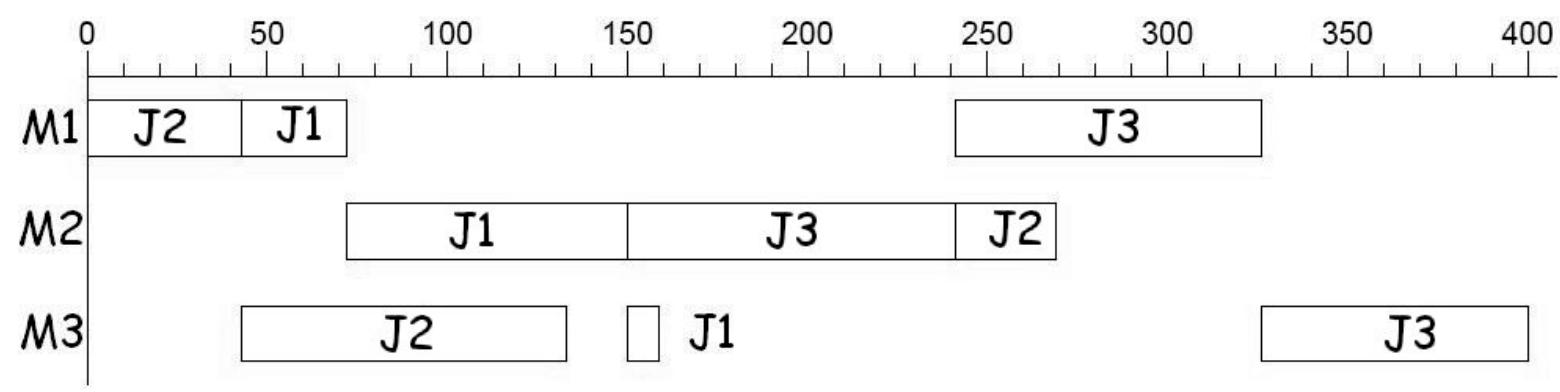

Figure 1: Gantt chart for the $3 \times 3$ instance. 
String:

((J2 M1 43) (J1 M1 29) (J1 M2 78) (J3 M2 91) (J2 M3 90) (J3 M1 85) (J2 M2 28) (J1 M3 9) (J3 M3 74))

Because the use of graphic Gantt charts in programming would be annoying, Gantt charts in numerical form are used. These charts are not as synoptic as graphical, but it is possible to comprehend all the important data from them. The Gantt chart in Fig. 1 looks in numerical form like this:

M1 (0 J2 43) (43 J1 72) (241 J3 326)

M2 (72 J1 150) (150 J3 241) (241 J2 269)

M3 (43 J2 133) (150 J1 159) (326 J3 400)

\section{GENETIC OPERATIONS}

Genetic operations are the driving force in genetic algorithms. Which operations are reasonable to use for solving a certain problem depends on the encoding method. The only genetic operation, which is independent from encoding, is selection. Selection is the most simple of all genetic operations. In our algorithm the tournament selection is used. Its purpose is to maintain the core of good solutions intact. This is done with transferring good solutions from one generation to the next one. Because selection does not change the organism (string), we have no problem with maintaining feasibility, which is not the case in all other genetic operations. The use of the crossover operation can be problematical in some cases. The crossover operation often produces infeasible offspring, which are difficult to repair.

The only genetic operation besides selection, which is used in our algorithm, is permutation. The permutation is based on switching operations inside the organism. The organism which will be permutated is chosen with the selection. When executing the permutation it is necessary to consider the precedence constrains. The permutation procedure is described and shown on our string from Table I:

a) A random operation is chosen from the string; let’s say (J2 M1 43).

String:

((J2 M1 43) (J1 M1 29) (J1 M2 78) (J3 M2 91) (J2 M3 90) (J3 M1 85) (J2 M2 28) (J1 M3 9) (J3 M3 74))

b) The left and the right border for the chosen operation have to be defined. Because the chosen operation belongs to job $J 2$, it is necessary to search for the first operation, left and right of the chosen operation, which belongs to job $J 2$. If the operation does not exist, the border is represented by the end or the beginning of the string. In our case, the right border is represented by the operation (J2 M3 90) and the left border is presented by the beginning of the string. In the string, the space between the borders is marked with square brackets.

String:

([(J2 M1 43) (J1 M1 29) (J1 M2 78) (J3 M2 91)] (J2 M3 90) (J3 M1 85) (J2 M2 28) (J1 M3 9) (J3 M3 74)) 
c) A random position between the brackets is chosen where the operation (J2 M1 43) is inserted; let us say in front of (J1 M2 78).

String:

((J1 M1 29) (J2 M1 43) (J1 M2 78) (J3 M2 91) (J2 M3 90) (J3 M1 85) (J2 M2 28) (J1 M3 9) (J3 M3 74))

This procedure randomly changes the chosen organism, which also changes the solution which the organism represents. Because there is often necessary to switch more than one operation in the organism, it is possible to repeat the whole procedure over and over.

\section{EVOLUTION PARAMETERS}

Evolution parameters are parameters, which influence the searching procedure of the genetic algorithm. If we want to obtain good solutions, the parameters have to be set wisely. These parameters are [15]:

- selection pressure,

- population size,

- amount of change, made by genetic operation,

- share of individual genetic operations in the next generation,

- number of generations (stopping criterion),

- number of independent civilizations.

The selection pressure defines what kind of solutions will be used in genetic operations. If the selection pressure is high, then only the best solutions will get the chance. If it is low, then also worse solutions are used. The higher the selection pressure, the higher the possibility that the search will end up in a local optimum. If it is too low, the search procedure examines insignificant solutions, which protracts the whole search.

When an organism is being modified, it is necessary to specify how much the genetic operation will change the organism. It is recommended that small and large changes are made to the organisms. This assures versatility in the population.

Each genetic operation must make a certain amount of organisms for the next generation. At least $10 \%$ of the next population has to be made with selection [16], so that the core of good solutions is maintained. All the other organisms are created with other genetic operations.

If the number of generations is multiplied with the size of the population, we get the number of organisms which have been examined during the search. When solving complex problems, the number of organisms is greater than in easier cases. The question is, should the search be executed with a small population and a lot of generations or opposite. In most cases a compromise is the best choice.

Because the search with genetic algorithms bases on random events, it is not necessary that good solutions are obtained in every civilization. In most cases the search stops in a local optimum. That is why it is necessary to execute multiple searches for a given problem.

\section{RESULTS}

The algorithm was tested on the notorious $10 \times 10$ and $20 \times 5$ instances which were proposed by Fisher and Thompson in 1963. Despite their small size, these two instances were very hard to solve. The $10 \times 10$ instance remained unsolved for nearly 25 years. 
Lestan, Brezocnik, Buchmeister, Brezovnik, Balic: Solving the Job-Shop Scheduling ...

Table II presents the $10 \times 10$ instance and Table III the $20 \times 5$ instance [17].

Table II: $10 \times 10$ instance.

\begin{tabular}{|c|c|c|c|c|c|c|c|c|c|c|}
\hline \multirow{2}{*}{ Jobs } & \multicolumn{10}{|c|}{ Operation sequence } \\
\cline { 2 - 11 } & 1. & 2. & 3. & 4. & 5. & 6. & 7. & 8. & 9. & 10. \\
\hline J1 & M1 29 & M2 78 & M3 9 & M4 36 & M5 49 & M6 11 & M7 62 & M8 56 & M9 44 & M10 21 \\
\hline J2 & M1 43 & M3 90 & M5 75 & M10 11 & M4 69 & M2 28 & M7 46 & M6 46 & M8 72 & M9 30 \\
\hline J3 & M2 91 & M1 85 & M4 39 & M3 74 & M9 90 & M6 10 & M8 12 & M7 89 & M10 45 & M5 33 \\
\hline J4 & M2 81 & M3 95 & M1 71 & M5 99 & M7 9 & M9 52 & M8 85 & M4 98 & M10 22 & M6 43 \\
\hline J5 & M3 14 & M1 6 & M2 22 & M6 61 & M4 26 & M5 69 & M9 21 & M8 49 & M10 72 & M7 53 \\
\hline J6 & M3 84 & M2 2 & M6 52 & M4 95 & M9 48 & M10 72 & M1 47 & M7 65 & M5 6 & M8 25 \\
\hline J7 & M2 46 & M1 37 & M4 61 & M3 13 & M7 32 & M6 21 & M10 32 & M9 89 & M8 30 & M5 55 \\
\hline J8 & M3 31 & M1 86 & M2 46 & M6 74 & M5 32 & M7 88 & M9 19 & M10 48 & M8 36 & M4 79 \\
\hline J9 & M1 76 & M2 69 & M4 76 & M6 51 & M3 85 & M10 11 & M7 40 & M8 89 & M5 26 & M9 74 \\
\hline J10 & M2 85 & M1 13 & M3 61 & M7 7 & M9 64 & M10 76 & M6 47 & M4 52 & M5 90 & M8 45 \\
\hline
\end{tabular}

Table III: $20 \times 5$ instance.

\begin{tabular}{|c|c|c|c|c|c|}
\hline \multirow{2}{*}{ Jobs } & \multicolumn{5}{|c|}{ Operation sequence } \\
\cline { 2 - 6 } & 1. & 2. & 3. & 4. & 5. \\
\hline J1 & M1 29 & M2 9 & M3 49 & M4 62 & M5 44 \\
\hline J2 & M1 43 & M2 75 & M4 69 & M3 46 & M5 72 \\
\hline J3 & M2 91 & M1 39 & M3 90 & M5 12 & M4 45 \\
\hline J4 & M2 81 & M1 71 & M5 9 & M3 85 & M4 22 \\
\hline J5 & M3 14 & M2 22 & M1 26 & M4 21 & M5 72 \\
\hline J6 & M3 84 & M2 52 & M5 48 & M1 47 & M4 6 \\
\hline J7 & M2 46 & M1 61 & M3 32 & M4 32 & M5 30 \\
\hline J8 & M3 31 & M2 46 & M1 32 & M4 19 & M5 36 \\
\hline J9 & M1 76 & M4 76 & M3 85 & M2 40 & M5 26 \\
\hline J10 & M2 85 & M3 61 & M1 64 & M4 47 & M5 90 \\
\hline J11 & M2 78 & M4 36 & M1 11 & M5 56 & M3 21 \\
\hline J12 & M3 90 & M1 11 & M2 28 & M4 46 & M5 30 \\
\hline J13 & M1 85 & M3 74 & M2 10 & M4 89 & M5 33 \\
\hline J14 & M3 95 & M1 99 & M2 52 & M4 98 & M5 43 \\
\hline J15 & M1 6 & M2 61 & M5 69 & M3 49 & M4 53 \\
\hline J16 & M2 2 & M1 95 & M4 72 & M565 & M3 25 \\
\hline J17 & M1 37 & M3 13 & M2 21 & M4 89 & M5 55 \\
\hline J18 & M1 86 & M2 74 & M5 88 & M3 48 & M4 79 \\
\hline J19 & M2 69 & M3 51 & M1 11 & M4 89 & M5 74 \\
\hline J20 & M1 13 & M2 7 & M3 76 & M4 52 & M5 45 \\
\hline
\end{tabular}


Table IV: Evolution parameters and results for the $10 \times 10$ and $20 \times 5$ instances.

\begin{tabular}{|l|c|c|}
\hline & $\mathbf{1 0 \times 1 0}$ & $\mathbf{2 0 \times 5}$ \\
\hline Population size & 200 & 200 \\
\hline Number of generations & 500 & 500 \\
\hline Number of independent populations & 100 & 100 \\
\hline Optimal solution & 935 & 1165 \\
\hline Best solution obtained & 941 & 1211 \\
\hline
\end{tabular}

As it can be seen from Table IV, the algorithm did not manage to find the optimal solution for the problem, but due its simplicity, the algorithm was able to obtain good solutions. Although both instances are made up of 100 operations, the solution space for the $20 \times 5$ instance is greater than that of the $10 \times 10$. The number of possible schedules $S$ (solutions) can be calculated with Eq. (1), where $m$ represents the number of machines and $n$ represents the number of jobs.

$$
S=(n !)^{m}
$$

So, for the first case we get: $S_{10 \times 10} \approx 4 \cdot 10^{65}$ possible schedules, and for the second case: $S_{20 \times 5} \approx 9 \cdot 10^{91}$ possible schedules.

The search was in both cases executed with the same evolution parameters. Because the algorithm is written in the LISP programming language [18] the search procedure took relatively long time. In the $10 \times 10$ case, the best obtained solution deviates $1.2 \%$ from the optimal solution. In the $20 \times 5$ case, the deviation was $4 \%$. The best result was in both cases obtained in only 1 run out of 100 runs. The search procedure often falls in a local optimum. When this happens, it is very unlikely that the search will proceed to a better solution, because a memory function is not present. This means that the quality of the final solution depends on the initial population and pure chance. This is why the search procedure has to be repeated several times for a specific problem.

\section{CONCLUSION}

It is obvious that job-shop scheduling plays an important role in the modern industry. The demanding market forces manufacturers to implement fast changes in their manufacturing space. These can be achieved only with the presence of a fast and powerful scheduler. Jobshop scheduling presents a hard combinatorial problem which is not easy to solve optimal. One of the methods for solving this stubborn problem is the genetic algorithm. Of course the performance of the algorithm depends on its design, which is linked with the experience and knowledge of the author. The truth is that not every evolution is a good evolution.

In this paper it is shown, that even a very simple evolution algorithm is capable of finding good solutions for the job-shop problem. Because the genetic algorithms are not well-suited for fine-tuning of solutions around optima the next step for this algorithm would be an upgrade to a hybrid algorithm. The algorithm, presented in this paper, only imitates natural evolution to find better solutions. Like in nature there are no instructions on how to achieve this. With a little heuristic help, the search procedure would become more efficient. The easiest way to do this would be to incorporate the local search procedure into the existing algorithm. With this improvement only the operations on critical paths would be switched in permutation. This would definitely speed up the search process and the search would not stop in a local optimum as easy as before. 


\section{REFERENCES}

[1] Sule, D. R. (2008). Production planning and industrial scheduling, CRC Press, Boca Raton

[2] T'kindt, V.; Billaut, J.-C. (2006). Multicriteria scheduling (Theory, models and algorithms), Springer-Verlag, Berlin

[3] Tasic, T.; Buchmeister, B.; Acko, B. (2007). The development of advanced methods for scheduling production processes, Strojniski vestnik - Journal of Mechanical Engineering, Vol. 53, No. 12, 844-857

[4] Buchmeister, B.; Kremljak, Z.; Pandza, K.; Polajnar, A. (2004). Simulation study on the performance analysis of various sequencing rules, International Journal of Simulation Modelling, Vol. 3, No. 2-3, 80-89

[5] Pinedo, M. L. (2005). Planning and scheduling in manufacturing and services, Springer Science+Business Media, New York

[6] Fowler, J. W.; Mönch, L.; Rose, O. (2006). Scheduling and simulation, Herrmann, J. W. (Editor), Handbook of production scheduling, Springer Science+Business Media, New York, 109-133

[7] Brucker, P. (2007). Scheduling algorithms, Springer-Verlag, Berlin

[8] Gen, M.; Cheng, R. (1997). Genetic algorithms and engineering design, John Wiley \& Sons, New York

[9] Zhang, C. Y.; Li, P.-G.; Rao, Y.-Q.; Guan, Z.-L. (2008). A very fast TS/SA algorithm for the job shop scheduling problem, Computers and Operations Research, Vol. 35, No. 1, 282-294

[10] Mitchell, T. M. (1997). Machine learning, McGraw-Hill, Singapore

[11] Haupt, R. L.; Haupt, S. E. (2004). Practical genetic algorithms, Wiley, New York

[12] Sung, K.; Anderson, J.; Bell, M. G. H. (1997). A genetic algorithm to optimise signal phasing in area traffic control, Second international conference on genetic algorithms in engineering systems, Glasgow, 404-408

[13] Tollazzi, T.; Lerher, T. (2008). An analysis of the influence of pedestrians` traffic flow on the capacity of a roundabout using the discrete simulation method, Strojniski vestnik - Journal of Mechanical Engineering, Vol. 52, No. 6, 359-379

[14] Cheng, R.; Gen, M.; Tsojimura, Y. (1996). A tutorial survey of job-shop scheduling problems using genetic algorithms - I: representation, Computers and Industrial Engineering, Vol. 30, No. 4, 983-997

[15] Mernik, M.; Crepinsek, M.; Zumer, V. (2003). Evolutionary algorithms (in Slovene), Faculty of Electrical Engineering and Computer Science, University of Maribor, Maribor

[16] Brezocnik, M. (2000). The use of genetic programming in intelligent manufacturing systems (in Slovene), Faculty of Mechanical Engineering, University of Maribor, Maribor

[17] Blazewicz, J.; Ecker, K. H.; Pesch, E.; Schmidt, G.; Weglarz, J. (2007). Handbook on scheduling, Springer-Verlag, Berlin

[18] Brezocnik, M. (2005). Guide for use of the Autolisp tool in AutoCAD environment (in Slovene), Faculty of Mechanical Engineering, University of Maribor, Maribor 